\title{
Reduced-Nicotine Cigarettes in Young Smokers: Impact of Nicotine Metabolism on Nicotine Dose Effects
}

\author{
Paul Faulkner', Dara G Ghahremani', Rachel F Tyndale ${ }^{2,3}$, Chelsea M Cox', Ari S Kazanjian', Neil Paterson ${ }^{4}$, \\ Shahrdad Lotfipour ${ }^{1,5}$, Gerhard S Hellemann', Nicole Petersen', Celia Vigil' and Edythe D London*,' \\ 'Department of Psychiatry and Biobehavioral Sciences, Semel Institute, University of California, Los Angeles, CA, USA; ${ }^{2}$ Department of \\ Pharmacology \& Toxicology, Campbell Family Mental Health Research Institute, Centre for Addiction \& Mental Health (CAMH), University of \\ Toronto, I King's College Circle, Toronto, ON, Canada; ${ }^{3}$ Department of Psychiatry, Campbell Family Mental Health Research Institute, Centre for \\ Addiction \& Mental Health (CAMH), University of Toronto, I King's College Circle, Toronto, ON, Canada; ${ }^{4}$ UCLA Semel Institute for Neuroscience \\ and Human Behavior, Los Angeles, CA, USA
}

\begin{abstract}
The use of cigarettes delivering different nicotine doses allows evaluation of the contribution of nicotine to the smoking experience. We compared responses of 46 young adult smokers to research cigarettes, delivering $0.027,0.110,0.231$, or 0.763 mg nicotine, and conventional cigarettes. On five separate days, craving, withdrawal, affect, and sustained attention were measured after overnight abstinence and again after smoking. Participants also rated each cigarette, and the nicotine metabolite ratio (NMR) was used to identify participants as normal or slow metabolizers. All cigarettes equally alleviated craving, withdrawal, and negative affect in the whole sample, but normal metabolizers reported greater reductions of craving and withdrawal than slow metabolizers, with dose-dependent effects. Only conventional cigarettes and, to a lesser degree, 0.763-mg nicotine research cigarettes increased sustained attention. Finally, there were no differences between ratings of lower-dose cigarettes, but the $0.763-\mathrm{mg}$ cigarettes and (even more so) conventional cigarettes were rated more favorably than lower-dose cigarettes. The findings indicate that smoking-induced relief of craving and withdrawal reflects primarily non-nicotine effects in slow metabolizers, but depends on nicotine dose in normal metabolizers. By contrast, relief of withdrawal-related attentional deficits and cigarette ratings depend on nicotine dose regardless of metabolizer status. These findings have bearing on the use of reduced-nicotine cigarettes to facilitate smoking cessation and on policy regarding regulation of nicotine content in cigarettes. They suggest that normal and slow nicotine metabolizers would respond differently to nicotine reduction in cigarettes, but that irrespective of metabolizer status, reductions to $<0.763 \mathrm{mg} /$ cigarette may contribute to temporary attentional deficits.
\end{abstract}

Neuropsychopharmacology (2017) 42, 1610-1618; doi:10.1038/npp.2017.18; published online 15 February 2017

\section{INTRODUCTION}

Tobacco smoking remains a leading contributor to preventable disease and death (Carter et al, 2015). Although nicotine is considered the main addictive chemical in cigarette smoke (Henningfield and Keenan, 1993), sensory stimuli (eg, taste, smell, and respiratory-tract sensations) and behavioral aspects of smoking also contribute (Rose and Behm, 1995). While transdermal nicotine helps some smokers quit (eg, Stead et al, 2008), most who use nicotine replacement therapies eventually relapse, partly because they miss the behavioral and sensory aspects of smoking (Rose et al, 2000). Smoking cigarettes that deliver less than conventional doses of nicotine (ie, $\sim 1 \mathrm{mg} /$ cigarette) reduce

*Correspondence: Dr ED London, Department of Psychiatry and Biobehavioral Sciences, Semel Institute, University of California Los Angeles, 760 Westwood Plaza, Los Angeles, CA 90025, USA, Tel: 310 825 0606, Fax: 310 825-08I2, E-mail: elondon@mednet.ucla.edu ${ }^{5}$ Current address: Department of Emergency Medicine \& Pharmacology, School of Medicine, University of California, Irvine, CA, USA.

Received 10 November 2016; revised 16 December 2016; accepted 19 January 2017; accepted article preview online 24 January 2017 nicotine dependence (Benowitz et al, 2007; Benowitz et al, 2012; Benowitz et al, 2015) and promote smoking cessation when used with transdermal nicotine (Hatsukami et al, 2013). Further, a trial of reduced-nicotine cigarettes delivering 0.05-1.2 mg nicotine in 780 smokers showed decreases in nicotine dependence and cigarette consumption at 6 weeks compared to baseline (Donny et al, 2015).

The Food and Drug Administration has the authority to set a standard for reduced nicotine content in cigarettes, but empirical data are needed to guide policy. Prior work has indicated that in 18-60 year-olds, smoking a cigarette delivering $<0.1 \mathrm{mg}$ nicotine alleviates craving and withdrawal less (albeit non-significantly) than a conventional cigarette (Buchhalter et al, 2001; Rose et al, 2000), but that cigarettes delivering $\sim 0.05 \mathrm{mg}$ nicotine provide relief as effectively as conventional cigarettes when smoked ad libitum for $90 \mathrm{~min}$ (Dallery et al, 2003; Tidey et al, 2013) or $4 \mathrm{~h}$ (Eid et al, 2005). Further, performance on the Rapid Visual Information Processing task (RVIP), a test of sustained attention, indicated greater discriminability between targets and non-targets (indicating increased sustained attention) after smoking a cigarette delivering $0.6 \mathrm{mg}$ 
nicotine $v s$ a cigarette delivering $0.05 \mathrm{mg}$ nicotine (Juliano et al, 2011), but performance before and after smoking was not compared.

It is important to consider how imposing a standard of reduced nicotine content in cigarettes might affect smokers. Young smokers are of particular interest because the transition from intermittent to daily smoking generally occurs before age 25 (Benowitz and Henningfield, 1994), and smoking cessation before this age avoids most long-term negative consequences of smoking (Doll et al, 2004). Young smokers smoke fewer cigarettes per day and are less nicotinedependent than older smokers, and therefore may respond differently to cigarettes delivering reduced nicotine (Benowitz and Henningfield, 1994).

Another important factor is nicotine metabolic rate, which is genetically determined (Tyndale and Sellers, 2001) and can be indexed by the NMR (Dempsey et al, 2004). When smokers were categorized as normal or slow metabolizers on the basis of an NMR criterion, normal metabolizers $(\geqslant 0.35)$ smoked more cigarettes/day (Chenoweth et al, 2014), but slow metabolizers $(<0.31)$ had a greater likelihood of 1 -week abstinence from smoking (Chenoweth et al, 2014). When smokers were divided into quartiles based on NMR, the fastest metabolizers had a greater likelihood of abstinence from smoking with bupropion treatment than the slowest metabolizers (Patterson et al, 2008). According to the same quartile system, the slowest metabolizers had lower nicotine dependence, reported less cigarette craving following overnight abstinence, and less drug liking after intravenous nicotine compared to the fastest metabolizers (Sofuoglu et al, 2012). While NMR (considered as a continuous variable) was not associated with decreases in nicotine dependence produced by smoking reduced-nicotine cigarettes over 6 months in one study (Bandiera et al, 2015), the interaction of nicotine dose and NMR on the acute effects of smoking are unknown.

We compared responses to smoking reduced-nicotine and conventional cigarettes in young, daily smokers. Cigarette craving, withdrawal and sustained attention were assessed after overnight abstinence, and again after smoking. Cigarette ratings, smoking topography and NMR were also evaluated. It was hypothesized that cigarettes delivering very low nicotine doses would alleviate craving and withdrawal as much as conventional cigarettes, but would be less effective at counteracting withdrawal-related deficits in sustained attention. Finally, because normal metabolizers report greater drug liking than slow metabolizers after receiving intravenous nicotine, it was hypothesized that normal metabolizers would display greater smoking-induced reductions in withdrawal symptoms than slow metabolizers, and that such group differences would increase with nicotine dose.

\section{MATERIALS AND METHODS}

Participants attended five testing sessions (3.14 \pm 2.01 days between sessions), after overnight $(\sim 12 \mathrm{~h})$ abstinence, verified by expired $\mathrm{CO}<10$ p.p.m. (measured using a coVita monitor, Haddonfield, NJ). They were required to have urine screens negative for abused drugs (including marijuana) at each session. Before and after smoking either a reduced-nicotine or their preferred-brand cigarette (counterbalanced, double-blind), participants had blood samples $(8 \mathrm{ml})$ drawn for plasma nicotine assay, rated withdrawal symptoms, and performed the RVIP (Foulds et al, 1996). After smoking, participants rated cigarette characteristics. At the start of the first test session a blood sample $(8 \mathrm{ml})$ was drawn for NMR determination. Between testing sessions, participants were allowed to smoke as usual.

\section{Participants}

Forty-six participants were recruited via online and print advertisements, attended an intake session, received a detailed explanation of study procedures (approved by the UCLA Institutional Review Board), gave written informed consent, and were screened for eligibility. Inclusion criteria were being $18-25$ years old and smoking $\geqslant 5$ cigarettes per day for $\geqslant 1$ year. Exclusion criteria were positive urine tests for abused drugs other than nicotine or marijuana, marijuana use $>8$ times/month or consuming alcohol $>15$ days/month, any Axis I psychiatric disorder other than nicotine dependence (Structured Clinical Interview for DSMIV, First et al, 1995), history of neurological injury, pregnancy, preferring menthol cigarettes, or using electronic cigarettes, cigars, snuff or chewing tobacco. Forty-six participants entered the study, and 40 completed all sessions.

\section{Cigarettes}

Research cigarettes, manufactured by 22nd Century Group Inc. (Spectrum - Clarence, NY), were provided by the National Institute on Drug Abuse, and delivered 0.027, 0.110, 0.231 , or $0.763 \mathrm{mg}$ nicotine, determined by the International Organization for Standardization Method. They were nonmentholated and did not vary in any other tobacco constituents or in ventilation. The mean nicotine dose of the preferred-brand cigarettes, estimated from the Federal Trade Commission (2007), was $1.1( \pm 0.24) \mathrm{mg}$.

\section{Smoking Topography}

A Clinical Research Support System topography monitor (Borgwaldt KC, Richmond, VA) was used to record the number of puffs per cigarette, and the average volume, intensity, and duration of each puff.

\section{Nicotine, Metabolites and NMR}

Fifteen participants provided blood samples before and after smoking, and another 14 provided samples after smoking only. Nicotine in plasma was assayed at Quest Diagnostics (Nichols Institute, Valencia, CA) by liquid chromatographytandem mass spectrometry after solid-phase extraction (Dempsey et al, 2004). The limit of quantification (LOQ) was $>2.0 \mathrm{ng}$ nicotine $/ \mathrm{ml}$, and the coefficient of variance at $10 \mathrm{ng} / \mathrm{ml}$ was $9.2 \%$. Nicotine concentrations below the LOQ were taken to be $1.4142 \mathrm{ng} / \mathrm{ml}$ (square root of the LOQ) as reported previously (Jacob et al, 2011).

The $3^{\prime}$-hydroxycotinine:cotinine ratio was determined using liquid chromatography-tandem mass spectrometry (Tanner et al, 2015). Participants were characterized as slow $(n=20)$ or normal $(n=21)$ metabolizers based on the 
median NMR of 0.35 , as reported previously (Chenoweth et al, 2014).

\section{Questionnaires}

The Fagerström test for Nicotine Dependence (FTND) was used (Fagerström, 2012), and the heaviness of smoking index (HSI), which is reportedly more predictive of success in maintaining abstinence from smoking (Borland et al, 2010), was derived from two questions on the FTND (time to first cigarette in the morning, and cigarettes/day). The ShiffmanJarvik Withdrawal Scale was used to obtain data on five subscales (craving, psychological, physiological, stimulation/ sedation and appetite; Shiffman and Jarvik, 1976). The positive and negative affect schedule was also used (Watson et al, 1988). The Cigarette Characteristics Questionnaire (Hatsukami et al, 2013b) was administered to evaluate ratings of flavor, strength, harshness, level of nicotine, and cigarette liking and disliking.

\section{Rapid Visual Information Processing Task}

The RVIP task was used to measure sustained attention (Foulds et al, 1996). Participants viewed a series of singledigit numbers $(75 / \mathrm{min}, 10 \mathrm{~min}$ ) on a computer screen and were to respond when seeing three consecutive odd or even numbers. Six targets/min were presented for $10 \mathrm{~min}$ with a response window of $800 \mathrm{~ms}$. The variable of interest was $\mathrm{A}^{\prime}$, a measure of discriminability between targets and nontargets, rather than the number of hits or reaction times, as the former is more sensitive to effects of nicotine (Foulds et al, 1996; Juliano et al, 2011; Leventhal et al, 2010).

\section{Statistical Analysis}

Statistical analyses were performed in the Statistical Package for Social Scientists (SPSS 21; IBM, Chicago, IL). Data from all 46 participants (40 completers) were included. For preand post-smoking measures, separate but parallel linear mixed models were employed, with relevant scores added as dependent variables, and time (pre-, post-smoking) and cigarette type (4 research cigarettes or preferred-brand) added as separate factors. NMR (slow, normal) and HSI were added as covariates to all models. Measures obtained postsmoking only were added to linear mixed models that were identical except that they did not contain time as a factor. All dependent variables, NMR and HSI met assumptions of normality.

Multiple comparisons corrections were performed as follows. For the Shiffman-Jarvik Withdrawal Scale and Cigarette Characteristics Questionnaire, Fisher's least significant difference method was used. Following omnibus tests (linear mixed models) on a 'total withdrawal' score and 'total cigarette rating' score, respectively, separate, parallel tests for each subscale were performed after the total score was deemed significant. The Bonferroni correction was used

Table I Participant Characteristics

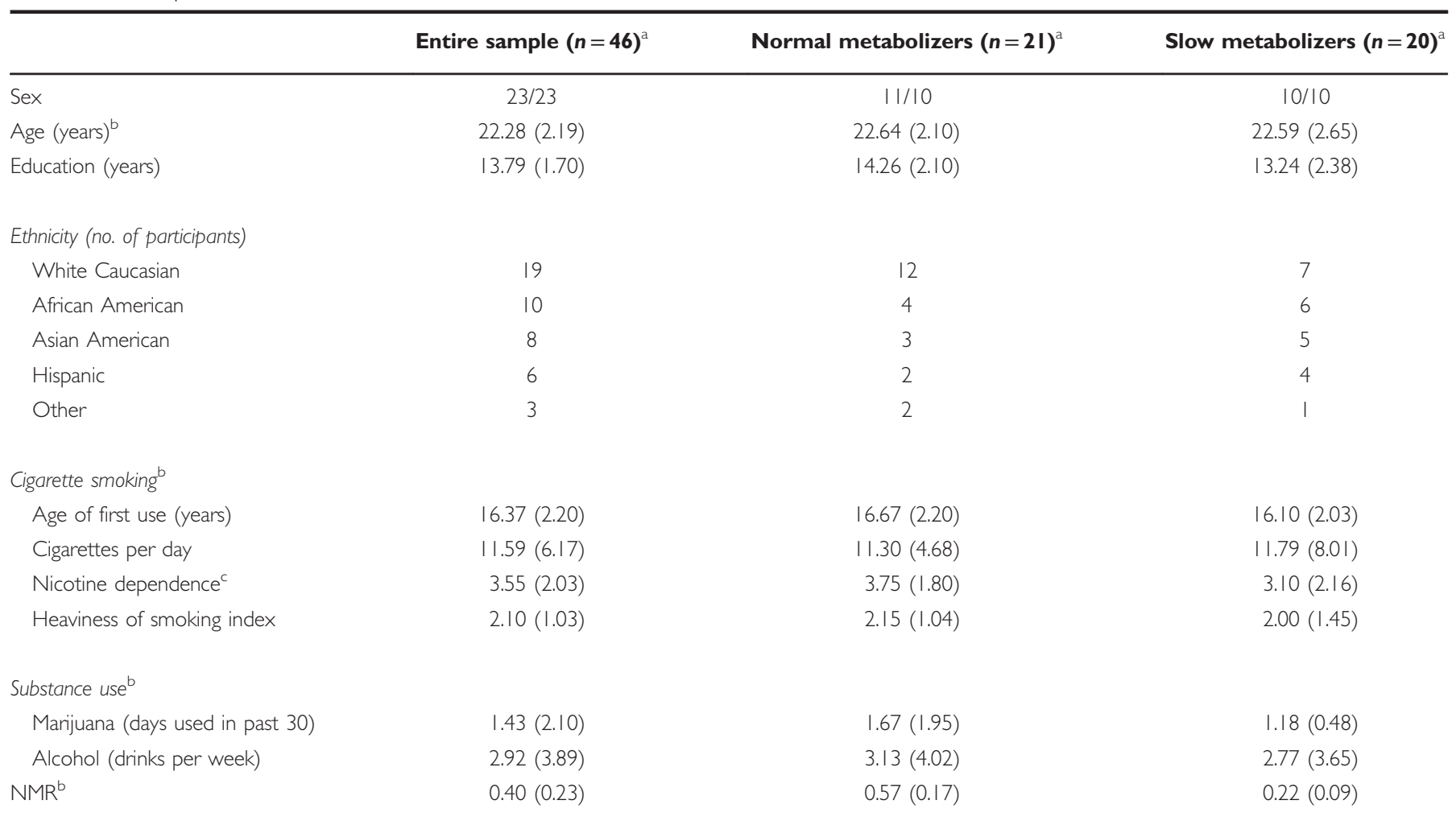

${ }^{a}$ Of the 46 participants, 40 completed all assessments, including five test sessions. One of the 6 noncompleters was a normal metabolizer, the other 5 were not tested for NMR.

bMean (SD).

'Determined using the FTND. 
for the two outcome measures in the Positive and Negative Affect Schedule (critical $p \leqslant 0.025$ ), and for data from the Shiffman-Jarvik Withdrawal Scale and the Positive and Negative Affect Schedule (critical $p$ 's $<0.025$ and 0.0125, respectively) because these two measures were not considered independent. For smoking topography, four variables (puffs/cigarette, average puff volume, intensity, and duration) were accounted for using the Bonferroni correction (critical $p \leqslant 0.0125$ ). Because sustained attention was considered independent of withdrawal and affect, no multiple comparisons correction was performed on data from the RVIP task.

\section{RESULTS}

\section{Participant Characteristics}

Forty-six daily smokers (23 women), mean age $22.28( \pm 2.19)$ years (51\% Caucasian), were included in the analyses (Table 1). Of these, 40 (20 women) completed all five testing sessions. Questionnaire and RVIP data were obtained from all participants. Due to blood-sampling difficulties, NMR was determined for all completing (and 1 non-completing) participants. The range of NMR values for the normal metabolizers was 0.38-1.07 (mean $=0.58, \pm 0.17$ ), and for slow metabolizers was $0.07-0.35$ (mean $=0.22$, \pm 0.092 ). Plasma nicotine concentration and smoking topography were measured in only 29 and 36 completing participants, respectively.

\section{Craving and Withdrawal}

Smoking reduced-nicotine cigarettes significantly reduced the total withdrawal score $(F(1,262)=62.984, p<0.001)$, but there was no effect of nicotine dose (no smoking-by-nicotine dose interaction; $\mathrm{F}(3,281)=0.370, p=0.774$ ) (Figure 1). Examination of the subscales on the Shiffman-Jarvik Withdrawal questionnaire revealed that smoking reduce-nicotine cigarettes relieved craving, psychological withdrawal, and appetite (all $p$ 's $<0.001$ ), with no effects of nicotine dose (all $p$ 's $>0.272)$.

Smoking the preferred-brand cigarette decreased total withdrawal $(\mathrm{F}(1,41)=11.064, p=0.002)$, but there was no difference between the effect of reduced-nicotine cigarettes and the preferred-brand cigarette $(\mathrm{F}(1,368)=0.027, p=0.869)$. Examination of the individual subscales revealed that the preferred-brand cigarette reduced craving, psychological withdrawal and appetite (all $p$ 's $<0.007$ ), but that there were no differences between the effects of reduced-nicotine cigarettes and the preferred-brand cigarette on any of these measures (all $p$ 's $>0.366$ ). No cigarette reduced scores on the 'Physiological' or 'Stimulation/Sedation' subscales of the ShiffmanJarvik Withdrawal Scale (all $p$ ’s $>0.146$ ).

NMR and effect of smoking on craving and withdrawal. Normal and slow metabolizers did not differ in terms of FTND $(\mathrm{F}(1,38)=0.935, p=0.340)$ or HSI scores $(F(1,38)=0.146, p=0.704)$ or cigarettes smoked per day $(\mathrm{F}(1,38)=0.073, p=0.788)$.

Compared to slow metabolizers, normal metabolizers reported greater decreases in total withdrawal due to smoking reduced-nicotine cigarettes (NMR by smoking interaction; $\mathrm{F}(1,270)=10.916, p<0.001)$. Post hoc tests revealed no metabolizer group differences on total withdrawal during abstinence $(F(1,36)=0.631, p=0.432)$ or after smoking $(F(1,36)=0.753, p=0.391)$. Examining the subscales of the Shiffman-Jarvik questionnaire revealed that compared to slow metabolizers, normal metabolizers reported greater decreases in craving $(\mathrm{F}(1,270)=6.042$, $p=0.015)$, appetite $(\mathrm{F}(1,270)=10.112, p<0.001)$, (Figure 1) and psychological withdrawal $(\mathrm{F}(1,270)=11.312, p<0.001)$ due to smoking reduced-nicotine cigarettes. Post hoc tests again revealed no metabolizer group differences on these measures before (all $p$ 's $>0.202$ ) or after smoking (all $p$ 's $>0.134$ ).

There was also a significant 3-way NMR-by-smoking-bynicotine dose interaction on total withdrawal $(\mathrm{F}(3,263)=6.427, p<0.001)$. Post hoc tests revealed normal metabolizers reported greater reductions in total withdrawal as the nicotine dose increased (significant smoking-by-dose interaction; $\mathrm{F}(3,128)=6.772, p<0.001)$, but slow metabolizers did not $F(3,128)=0.429, p<0.733)$. Examining each subscale independently, there was a significant 3-way NMR-bysmoking-by-nicotine dose interaction on cigarette craving $(\mathrm{F}(3,263)=11.090, p<0.001)$, and appetite $(F(3,263)=4.156$, $p=0.020)$ but not psychological withdrawal $(F(3,263)=0.821$, $p=0.483)$. Post hoc tests revealed that normal metabolizers reported greater reductions in craving and appetite as nicotine dose increased (all $p$ 's $<0.003$ ), but slow metabolizers did not (all $p$ 's $>0.331$ ). No such interaction effects were observed for any other withdrawal measure (all $p$ 's $>0.274$ ).

Regarding the preferred-brand cigarette, compared to slow metabolizers, normal metabolizers reported greater smokinginduced decreases in total withdrawal $(\mathrm{F}(3,56)=4.157$, $p=0.010)$, and the preferred-brand cigarette produced greater reductions in total withdrawal than the reducednicotine cigarettes in normal metabolizers more than in slow metabolizers (significant 3-way NMR-by-smoking-by-cigarette type; $\mathrm{F}(1,345)=6.171, p=0.018)$. This pattern of results was also observed for the craving and appetite subscales of the Shiffman-Jarvik Withdrawal scale (all $p$ 's $<0.022$ ), but not other subscales (all $p$ 's $>0.415$ ).

\section{Affect}

Smoking reduced-nicotine cigarettes increased positive affect $(\mathrm{F}(1,262)=25.134, p<0.001)$ and reduced negative affect $(\mathrm{F}(1,262)=28.371, p<0.001)$, with no nicotine dose effect on positive (no smoking-by-nicotine dose interaction; $\mathrm{F}(3,281)=0.446, \quad p=0.721) \quad$ or negative affect $(\mathrm{F}(3,281)=0.373, p=0.772)$ (Figure 2). Further, participants' preferred-brand cigarette increased positive affect $(\mathrm{F}(1,41)=10.797, p=0.002)$ and decreased negative affect $(F(1,41)=18.283, p<0.001)$, but there were no differences between the effects of the reduced-nicotine and preferredbrand cigarettes on either positive $(\mathrm{F}(1,368)=0.881$, $p=0.349)$ or negative affect $(\mathrm{F}(1,368)=0.289, p=0.591)$.

NMR and effect of smoking on affect. There was no main effect of NMR on alterations in positive or negative affect due to smoking reduced-nicotine cigarettes or the preferredbrand cigarette (all $p$ 's $>0.464$ ). There were also no significant 3-way NMR-by-smoking-by-nicotine dose interactions on either measure (both $p$ 's $>0.426$ ), and no metabolizer group effects on differences between the 
Before Smoking

After Smoking

Whole Group
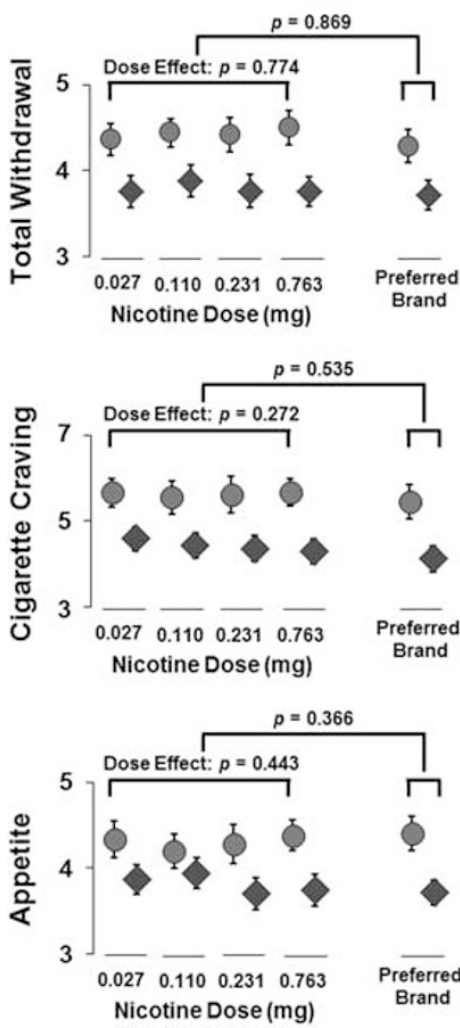

Normal Metabolizers
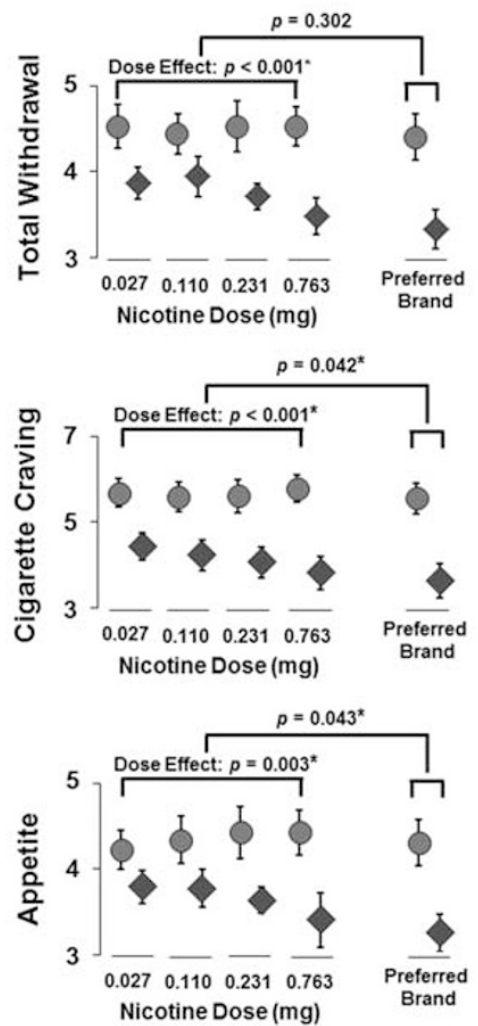

Slow Metabolizers
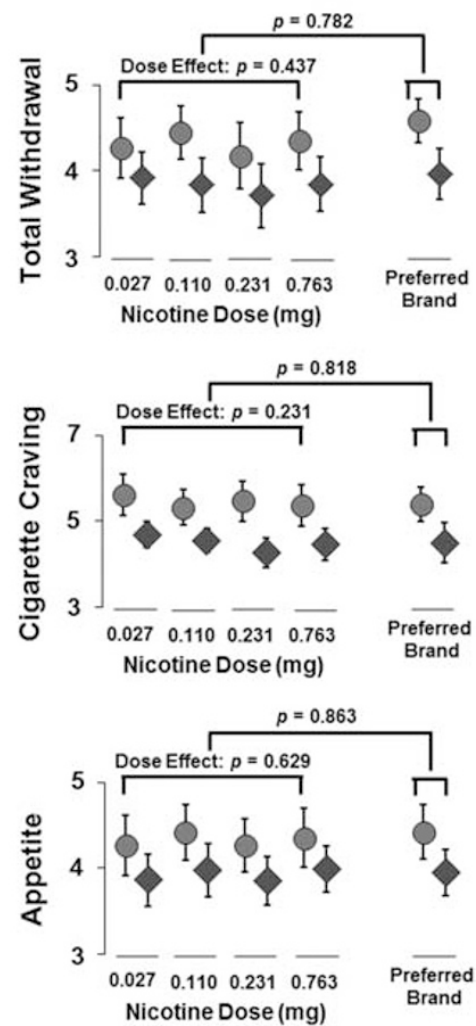

Figure I Effects of reduced-nicotine and preferred-brand cigarettes on total withdrawal, cigarette craving, and appetite. Total withdrawal cigarette craving and appetite on the Shiffman-Jarvik Withdrawal Scale (scale 0-7) as shown in the entire sample and subsamples characterized as normal metabolizers or slow metabolizers. Linear mixed models revealed that all cigarettes reduced scores on these measures. However, there were no significant main effects of the nicotine dose delivered by the reduced-nicotine cigarettes, and no difference between the effect of the reduced-nicotine cigarettes and the preferred-brand cigarette in the entire sample. There was a significant effect of nicotine dose delivered by the reduced-nicotine cigarette on total withdrawal, craving, and appetite in normal metabolizers but not slow metabolizers. The preferred-brand cigarettes decreased craving and appetite (but not total withdrawal) more than the reduced-nicotine cigarettes (all doses combined) in normal but not slow metabolizers. Error bars denote \pm SEM, * $\alpha$ levels set at $<0.05$.

reduced-nicotine cigarettes and preferred-brand cigarettes on affect (both $p$ 's $>0.642$ ).

\section{Sustained Attention}

There was no effect of session on $\mathrm{A}^{\prime}(\mathrm{F}(9,362)=0.920 p=0.508)$, indicating that there was no practice effect on task performance (Figure 3). Smoking the reduced-nicotine cigarettes (combined) did not improve $\mathrm{A}^{\prime}(F(1,285)=2.236, p=0.136)$, but there was a significant smoking-by-nicotine dose interaction on $\mathrm{A}^{\prime}$ $(F(3,280)=2.283, p=0.028)$. Post hoc tests revealed that the 0.763 -mg cigarette increased $\mathrm{A}^{\prime}(\mathrm{F}(1,40)=4.982, p=0.030)$, whereas the three lower-dose cigarettes did not (all $p$ 's $>0.730$ ). Smoking the $0.763-\mathrm{mg}$ cigarette increased $\mathrm{A}^{\prime}$ more than the three lower-dose cigarettes combined $(F(1,284)=4.751$, $p=0.027)$. Finally, smoking the preferred-brand cigarette increased $\mathrm{A}^{\prime}(\mathrm{F}(1,40)=5.918, p=0.020)$, and did so more than the 0.763 -mg cigarette $(\mathrm{F}(1,120)=3.643, p=0.015)$.

NMR and effect of smoking on sustained attention. There was no main effect of NMR on increases in $A^{\prime}$ due to smoking reduced-nicotine cigarettes or the preferred-brand cigarette (both $p$ 's $>0.443$ ) (Figure 3). There were no significant 3-way NMR-by-smoking-by-nicotine dose interactions on $\mathrm{A}^{\prime}(F(3,263)=0.752 p=0.645)$, and no effect of metabolizer group on differences between the effects of the reduced-nicotine cigarettes and preferred-brand cigarettes $(\mathrm{F}(4,200)=1.109, p=0.353)$.

\section{Cigarette Characteristics}

There was a significant effect of nicotine dose on total ratings of the reduced-nicotine cigarettes $(\mathrm{F}(3,110)=6.705$, $p<0.001)$. A post hoc test revealed that this effect was due to higher rating for the $0.763-\mathrm{mg}$ cigarette than cigarettes at the three lower doses $(F(1,121)=15.026, p<0.001)$; total ratings of the three lower-dose cigarettes did not differ $(\mathrm{F}(2,80)=0.900, p=0.411)$. Total rating scores were higher for the preferred-brand cigarette compared to the reducednicotine cigarettes combined $(\mathrm{F}(1,91)=31.998), p<0.001)$, and compared to the $0.763-\mathrm{mg}$ research cigarette $(\mathrm{F}(1,21)=30.173, p<0.001)$.

Examining each subscale independently, results revealed a significant effect of nicotine dose on ratings of the 
Whole Group
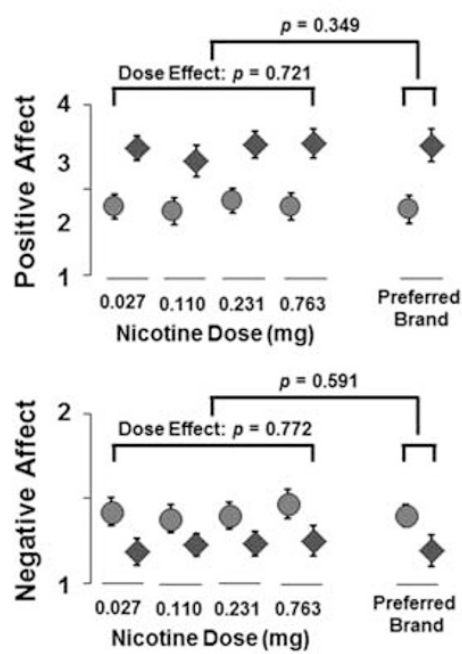

Normal Metabolizers
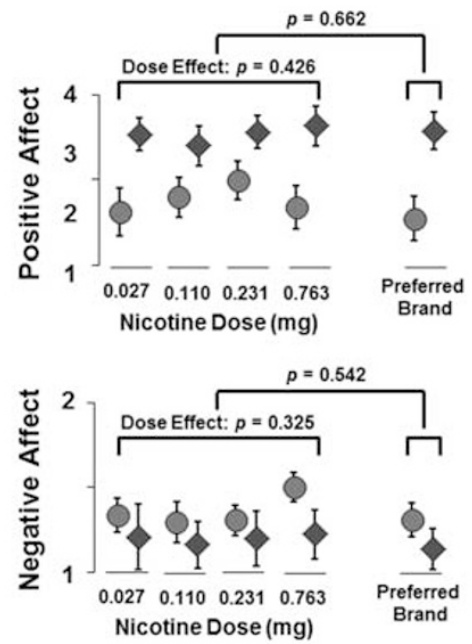

Slow Metabolizers
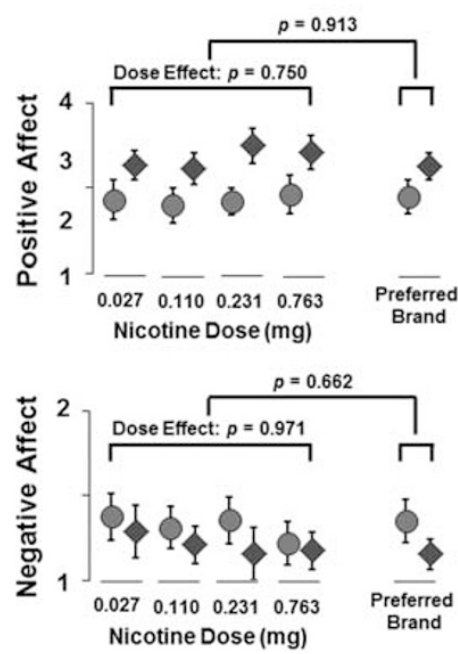

Figure 2 Effects of reduced-nicotine and preferred-brand cigarettes on positive and negative affect. Positive affect and negative affect as shown for the entire sample, normal metabolizers and slow metabolizers. Linear mixed models revealed that all cigarettes increased positive affect and decreased negative affect. There were no effects of the nicotine dose of the research cigarettes, and no difference between the effect of reduced-nicotine cigarettes and the preferred-brand cigarette on either measure. Error bars denote \pm SEM, $\alpha$ levels set at $<0.05$.

\section{Research Cigarette \\ Preferred Brand Cigarette}

Whole Group

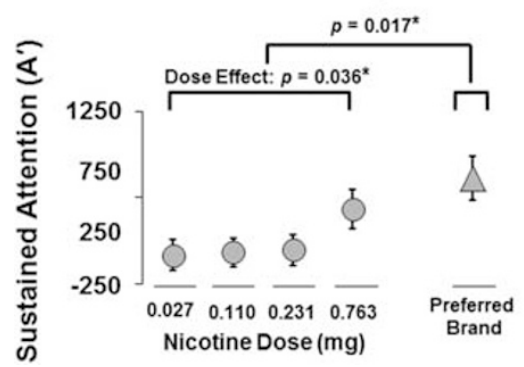

Normal Metabolizers

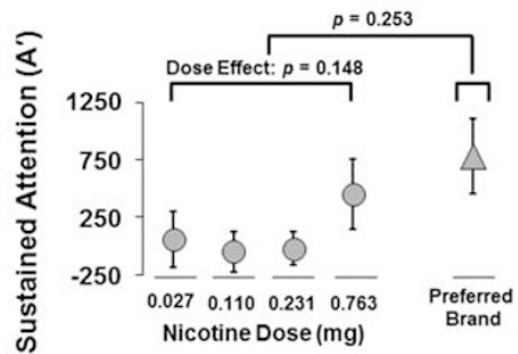

Slow Metabolizers

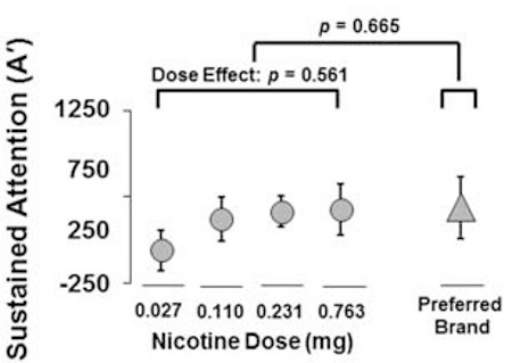

Figure 3 Effect of nicotine dose on sustained attention. Mean smoking-induced change scores in $A^{\prime}$ scores in the entire sample, normal and slow metabolizers. In the whole group, linear mixed models revealed significant main effects of smoking the preferred-brand cigarette and the 0.763 -mg nicotine research cigarette, but no main effects of smoking research cigarette delivering $\leqslant 0.23 \mathrm{I} \mathrm{mg}$ nicotine. Further, there were significant differences between the effect of the preferred-brand cigarette and reduced-nicotine cigarettes (all doses combined), between the preferred-brand cigarette and 0.763-mg cigarette, and between the $0.763-\mathrm{mg}$ research cigarette and the three lower dose cigarettes, but not between the three lower dose cigarettes. In normal (but not slow) metabolizers, preferred-brand cigarettes improved sustained attention, and did so more than the reduced-nicotine cigarettes (all doses combined). No other significant main or interaction effects were observed in either metabolizer subgroup. Error bars denote \pm SEM, * $\alpha$ levels set at $<0.05$.

reduced-nicotine cigarettes in terms of strength, perceived nicotine content, liking and disliking (all $p$ 's $<0.008$; Figure 4), and a trend towards such an effect on ratings of flavor $(\mathrm{F}(1,125)=2.465, p=0.065)$. Post hoc tests revealed that these effects were due to participants rating the $0.763-\mathrm{mg}$ cigarette more favorably on all of these measures (all $p$ 's $<0.001$ ) whereas ratings of the three lower-dose cigarettes did not differ (all $p$ 's $>0.678$ ). The preferred-brand cigarette was rated more favorably on all characteristics (all $p$ 's $<0.001$ ) compared to the reduced-nicotine cigarettes (combined), and compared to the $0.763-\mathrm{mg}$ research cigarette (all $p$ 's $<0.001$ ).
NMR and cigarette ratings. There were no main effects of NMR on ratings of either the reduced-nicotine cigarettes or the preferred-brand cigarette (all $p$ 's $>0.195$ ). Further, there were no significant 3-way NMR-by-smoking-by-nicotine dose interactions on such ratings (all $p$ 's $>0.338$ ), and no effect of metabolizer group on differences between the ratings of the reduced-nicotine cigarettes and preferred-brand cigarettes $(p>0.247)$.

\section{Heaviness of Smoking Index}

Participants with larger HSI scores reported greater decreases in total withdrawal, craving, and psychological withdrawal after 
Whole Group

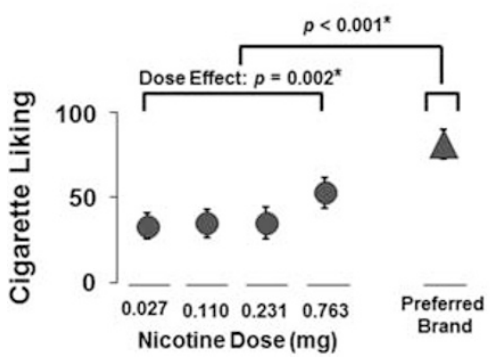

Normal Metabolizers

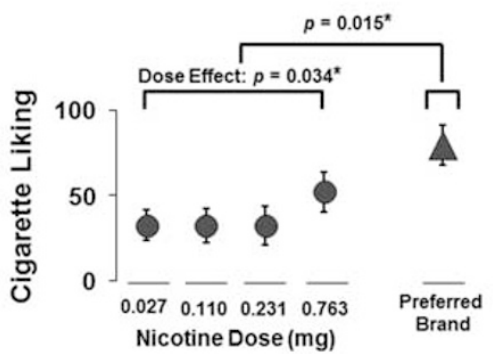

Slow Metabolizers

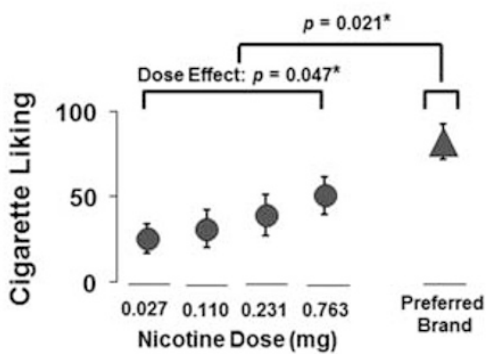

Figure 4 Effect of nicotine dose on cigarette liking. Cigarette liking in the entire sample, normal, and slow metabolizers. In the whole group, normal and slow metabolizers, there were significant differences between liking of the preferred-brand cigarette and reduced-nicotine cigarettes (all doses combined), between liking of the preferred-brand cigarette and 0.763-mg cigarette, and between liking of the 0.763-mg research cigarette and the three lower dose cigarettes, but not between liking of the three lower dose cigarettes. Error bars denote \pm SEM, * $\alpha$ levels set at $<0.05$.

smoking both reduced-nicotine cigarettes and preferred-brand cigarettes (HSI-by-smoking interactions; all $p$ 's $<0.002$ ). Post hoc tests revealed that HSI score was positively associated with total withdrawal $(\mathrm{F}(1,42)=7.050, p=0.011)$, craving $(\mathrm{F}(1,42)=18.635, p<0.001)$, and psychological withdrawal $(\mathrm{F}(1,42)=5.065, p=0.030)$ during abstinence, but not after smoking (all $p$ 's $>0.267$ ). HSI score was not associated with effects of reduced-nicotine cigarettes or the preferred-brand cigarette on sustained attention or cigarette ratings (all $p$ 's $>0.237$ ), nor did it modulate the effect of nicotine dose on any measure (all $p$ 's $>0.243$ ).

\section{Smoking Topography}

There were no differences between the reduced-nicotine cigarettes, or between reduced-nicotine cigarettes and the preferred-brand cigarette, in puff count, average volume, intensity, or duration (all $p$ 's $>0.365$ ). Moreover, there were no differences between normal and slow metabolizers on any such measures when smoking reduced-nicotine cigarettes or the preferred-brand cigarette (all $p$ 's $>0.237$ ).

\section{Plasma Nicotine}

Blood samples were drawn on average $15.16( \pm 7.36) \mathrm{min}$ after smoking. Pre-smoking plasma nicotine levels (after overnight abstinence) were obtained from 4 normal metabolizers and 3 slow metabolizers, and the values did not differ (slow metabolizers mean $(\mathrm{SD})=3.37$ (2.34), range 1.41-8.70, normal metabolizers mean $(\mathrm{SD})=3.03$ (2.49), range $1.41-9.90 ; \mathrm{F}(1,6)=0.192, p=0.679)$. Smoking reducednicotine cigarettes increased plasma nicotine levels $(\mathrm{F}(1,120)=15.191, p<0.001)$; and there was a significant smoking-by-nicotine dose interaction $(\mathrm{F}(3,100)=5.565$, $p=0.001)$ - plasma nicotine increased with nicotine dose. Smoking the preferred-brand cigarette also increased plasma nicotine levels $(F(1,9)=16.321, p=0.003)$, more so than the reduced-nicotine cigarettes $(\mathrm{F}(2,18)=4.560, p=0.025)$. There were no differences between the plasma nicotine levels of slow and normal metabolizers after smoking reduced-nicotine cigarettes $(\mathrm{F}(2,123)=0.537, p=0.586)$ or preferred-brand cigarettes $(\mathrm{F}(2,9)=0.921, p=0.432)$.

\section{DISCUSSION}

This study of young smokers provides the first evidence that smoking-induced reductions in craving and withdrawal depend on nicotine dose in normal but not slow metabolizers. Improvement in sustained attention during acute abstinence and ratings of cigarette characteristics both depend on nicotine dose, regardless of metabolizer status. These findings have bearing on the use of reduced-nicotine cigarettes to facilitate smoking cessation and on policy regarding regulation of nicotine content in cigarettes.

The reasons for different effects of smoking in normal $v s$ slow metabolizers have been investigated using brain imaging. Compared to slow metabolizers, normal metabolizers exhibit greater smoking cue-induced activation in the amygdala, hippocampus, left caudate, right insula, and cingulate cortex during satiety (Tang et al, 2012), but greater smoking cue-induced activation in the left caudate, left inferior frontal gyrus, and left frontal pole during abstinence (Falcone et al, 2015).

However, no previous study has examined differences between normal and slow metabolizers in the effects of smoking on craving and withdrawal. That smoking-induced reductions in craving and withdrawal in slow metabolizers were not dependent on nicotine dose indicates that such reductions may be related to the sensory and behavioral aspects of smoking (Rose and Levin, 1991), which may trigger dopamine release in the nucleus accumbens (Schultz, 1998). However, that relief of craving and withdrawal was nicotine-dose dependent in normal metabolizers indicates that such relief may be related to the nicotine-dosedependent effects on both dopamine release in the ventral striatum (Brody et al, 2009a) and nicotinic receptor occupancy (Brody et al, 2009b). Importantly, in both normal and slow metabolizers, effects of smoking cigarettes delivering very low doses of nicotine may also involve non-nicotine constituents of cigarette smoke, such as the harmala alkaloids, which inhibit monoamine oxidase A, and increase the reinforcing effects of low, but not high, doses of nicotine in rats (Smith et al, 2016).

This report provides evidence that cigarettes delivering $0.763 \mathrm{mg}$ nicotine (a dose similar to conventional doses) 
significantly alleviate withdrawal-related deficits in sustained attention, but that cigarettes delivering substantially lower doses ( $\leqslant 0.231 \mathrm{mg}$ nicotine) do not, which suggests a threshold for nicotine dose. Successful performance on the RVIP task is related to activation in the prefrontal cortex and parietal cortex, as well as the insula and anterior cingulate cortex (Lawrence et al, 2003), the latter two regions having relatively high densities of nicotinic acetylcholine receptors (Picard et al, 2013). Thus, improvements in sustained attention could reflect effects of nicotine in the insula and/ or anterior cingulate cortex.

Information from this study can guide policy regarding how a reduction in nicotine content of cigarettes may affect young smokers. Such a reduction may have a greater effect on normal metabolizers than on slow metabolizers, but cigarettes delivering very low doses of nicotine can alleviate craving and withdrawal to a similar extent in both metabolizer groups. Reducing the nicotine yield of cigarettes below $0.763 \mathrm{mg}$ may produce transient difficulties in concentrating when young smokers switch from conventional cigarettes, regardless of nicotine metabolism rate. However, difficulty concentrating is a symptom of nicotine withdrawal that typically peaks within 2-3 days and wanes after $~ 4-6$ days (Hughes, 2007; Hughes and Hatsukami, 1986). That there were no differences in ratings for cigarettes delivering $\leqslant 0.231 \mathrm{mg}$, but that the 0.763 mg cigarette was rated as more favorable with the preferredbrand cigarette rated more favorably still, indicates that reducing the nicotine content of cigarettes to $<0.763 \mathrm{mg}$ may reduce the reinforcement provided by smoking, and additional reductions below $0.231 \mathrm{mg}$ would not reduce reinforcement further.

This study has some limitations. It involved only young smokers, who typically smoke fewer cigarettes/day than older smokers (CDC, 2016), and are slower metabolizers of nicotine because slower metabolizers typically quit smoking earlier than normal metabolizers (Ho et al, 2009). Therefore, the results may not be widely generalizable. Also, pre-smoking plasma nicotine levels were not obtained for most participants, limiting ability to assess the effect of the smoking-induced nicotine boost. Finally, a comparison of reduced-nicotine cigarettes to research cigarettes that deliver a conventional dose of nicotine would give a clearer understanding of the mediating effect of nicotine dose on responses to smoking.

\section{FUNDING AND DISCLOSURE}

This research was supported, in part, by grants from the National Institute on Drug Abuse (R01DA036487), the Canada Research Chair in Pharmacogenomics (RFT), a NARSAD Young Investigator Grant (21517, SL) and endowments from the Thomas $\mathrm{P}$ and Katherine $\mathrm{K}$ Pike Chair in Addiction Studies and the Marjorie M Greene Trust (EDL). As principal investigator of the project, Dr. London, takes responsibility for the integrity of the data and the accuracy of the data analysis. The authors declare no conflict of interest.

\section{ACKNOWLEDGMENTS}

We thank Dr Kyoji Okita for insightful comments and for his role in the preparation of the graphs contained in this manuscript. We also thank Dr Elliot Stein, who provided a version of the RVIP task, which was adapted for use in this study.

\section{REFERENCES}

Bandiera FC, Ross KC, Taghavi S, Delucchi K, Tyndale RF, Benowitz NL (2015). Nicotine dependence, nicotine metabolism, and the extent of compensation in response to reduced nicotine content cigarettes. Nicotine Tob Res 17: 1167-1172.

Benowitz NL, Hall SM, Stewart S, Wilson M, Dempsey D, Jacob P (2007). Nicotine and carcinogen exposure with smoking of progressively reduced nicotine content cigarette. Cancer Epidemiol Biomarkers Prev 16: 2479-2485.

Benowitz NL, Dains KM, Hall SM, Stewart S, Wilson M, Dempsey $\mathrm{D}$ et al (2012). Smoking behavior and exposure to tobacco toxicants during 6 Months of smoking progressively reduced nicotine content cigarettes. Cancer Epidemiol Biomarkers Prev 21: 761-769.

Benowitz NL, Nardone N, Dains KM, Hall SM, Stewart S, Dempsey $\mathrm{D}$ et al (2015). Effect of reducing the nicotine content of cigarettes on cigarette smoking behavior and tobacco smoke toxicant exposure: 2-year follow up. Addiction 110: 1667-1675.

Benowitz NL, Henningfield JE (1994). Establishing a nicotine threshold for addiction. The implications for tobacco regulation. $N$ Engl J Med 331: 123-125.

Borland R, Yong HH, O'Connor RJ, Hyland A, Thompson ME (2010). The reliability and predictive validity of the Heaviness of Smoking Index and its two components: findings from the International Tobacco Control Four Country study. Nicotine Tob Res 12: S45-S50.

Brody AL, Mandelkern MA, Olmstead RE, Allen-Martinez Z, Scheibal D, Abrams AL et al (2009a). Ventral striatal dopamine release in response to smoking a regular vs a denicotinized cigarette. Neuropsychopharmacology 34: 282-289.

Brody AL, Mandelkern MA, Costello MR, Abrams AL, Scheibal D, Farahi J et al (2009b). Brain nicotinic acetylcholine receptor occupancy: effect of smoking a denicotinized cigarette. Int $J$ Neuropsychopharmacol 12: 305-316.

Buchhalter AR, Schrinel L, Eissenberg T (2001). Withdrawalsuppressing effects of a novel smoking system: comparison with own brand, not own brand, and de-nicotinized cigarettes. Nicotine Tob Res 3: 111-118.

Carter BD, Freedman ND, Jacobs EJ (2015). Smoking and mortality-beyond established causes. N Engl J Med 372: 2170.

Centers for Disease Control and Prevention. Cigarette Smoking Among Adults-United States, 2005-2015. Morbidity and Mortality Weekly Report 2016; 65(44): 1205-1211.

Chenoweth MJ, Novalen M, Hawk LW, Schnoll RA, George TP, Cinciripini $\mathrm{P}$ et al (2014). Known and novel sources of variability in the nicotine metabolite ratio in a large sample of treatment-seeking smokers. Cancer Epidemiol Biomarkers Prev 23: 1773-1782.

Dallery J, Houtsmuller EJ, Pickworth WB, Stitzer ML (2003). Effects of cigarette nicotine content and smoking pace on subsequent craving and smoking. Psychopharmacology (Berl) 165: 172-180.

Dempsey D, Tutka P, Jacob P, Allen F, Schoedel K, Tyndale RF et al (2004). Nicotine metabolite ratio as an index of cytochrome P450 2A6 metabolic activity. Clin Pharmacol Ther 76: 64-72.

Doll R, Peto R, Boreham J, Sutherland I (2004). Mortality in relation to smoking: 50 years' observations on male British doctors. BMJ 328: 1519.

Donny EC, Denlinger RL, Tidey JW, Koopmeiners JS, Benowitz NL, Vandrey RG et al (2015). Randomized trial of reduced-nicotine standards for cigarettes. N Engl J Med 373: 1340-1349.

Eid NC, Fant RV, Moolchan ET, Pickworth WB (2005). Placebo cigarettes in a spaced smoking paradigm. Pharmacol Biochem Behav 81: 158-164. 
Fagerström K (2012). Determinants of tobacco use and renaming the FTND to the Fagerström Test for Cigarette Dependence. Nicotine Tob Res 14: 75-78.

Falcone M, Cao W, Bernardo L, Tyndale RF, Loughead J, Lerman C (2015). Brain responses to smoking cues differ based on nicotine metabolism rate. Biol Psychiatry 80: 190-197.

Federal Trade Commission (2007). Tar, Nicotine and Carbon Monoxide Reports for the years 1999-2005. Available at https:// web.archive.org/web/20131104130634/http:/www.ftc.gov/foia/fre quentrequests/tarnicotineletter.pdf.

First MB, Spitzer RL, Gibbon M, Williams JB (1995). The structured clinical interview for DSM-IV axis I disorders (SCID-IP). American Psychiatric Press: Washington, DC, USA.

Foulds J, Stapleton J, Swettenham J, Bell N, McSorley K, Russell MA (1996). Cognitive performance effects of subcutaneous nicotine in smokers and never-smokers. Psychopharmacology (Berl) 127: 31-38.

Hatsukami DK, Hertsgaard LA, Vogel RI, Jensen JA, Murphy SE, Hecht SS et al (2013). Reduced nicotine content cigarettes and nicotine patch. Cancer Epidemiol Biomarkers Prev 22: 1015-1024.

Hatsukami DK, Zhang Y, O'Connor RJ, Severson HH (2013b). Subjective responses to oral tobacco products: scale validation. Nicotine Tob Res 15: 1259-1264.

Henningfield JE, Keenan RM (1993). Nicotine delivery kinetics and abuse liability. J Consult Clin Psychol 61: 743-750.

Ho MK, Mwenifumbo JC, Al Koudsi N, Okuyemi KS, Ahluwalia JS, Benowitz NL et al (2009). Association of nicotine metabolite ratio and CYP2A6 genotype with smoking cessation treatment in African-American light smokers. Clin Pharmacol Therapeutics 85: 635-643.

Hughes JR (2007). Effects of abstinence from tobacco: valid symptoms and time course. Nicotine Tob Res 9: 315-327.

Hughes JR, Hatsukami D (1986). Signs and symptoms of tobacco withdrawal. Arch Gen Psychiatry 43: 289-294.

Jacob P, Abu Raddaha AH, Dempsey D, Havel C, Peng M, Yu L et al (2011). Nicotine, carbon monoxide, and carcinogen exposure after a single use of a waterpipe. Cancer Epidemiol Biomarkers Prev 20: 2345-2353.

Juliano LM, Fucito LM, Harrell PT (2011). The influence of nicotine dose and nicotine dose expectancy on the cognitive and subjective effects of cigarette smoking. Exp Clin Psychopharmacol 19: 105-115.

Lawrence NS, Ross TJ, Hoffman R, Garavan H, Stein EA (2003). Multiple neuronal networks mediate sustained attention. J Cogn Neurosci 15: 1028-1038.

Leventhal AM, Waters AJ, Moolchan ET, Hesihman SJ, Pickworth WB (2010). A quantitative analysis of subjective, cognitive and physiological manifestations of the acute tobacco abstinence syndrome. Addictive Behav 35: 1120-1130.

Patterson F, Schnoll RA, Wileyto EP, Pinto A, Epstein LH, Shields PG et al (2008). Toward personalized therapy for smoking cessation: a randomized placebo-controlled trial of bupropion. Clin Pharmacol Ther 84: 320-325.

Picard F, Sadaghiani S, Leroy C, Courvoisier DS, Maroy R, Bottlaender M (2013). High density of nicotinic receptors in the cingulo-insular network. Neuroimage 79: 42-51.

Rose JE, Behm FM (1995). There is more to smoking than the CNS effects of nicotine. In: Clarke PBS, Quik M, Adlkofer F, Thurau K (eds). Effects of Nicotine on Biological Systems II. Birkhäuser Basel: Basel, Switzerland, pp 239-246.

Rose JE, Behm FM, Westman EC, Johnson M (2000). Dissociating nicotine and nonnicotine components of cigarette smoking. Pharmacol Biochem Behav 67: 71-81.

Rose JE, Levin ED (1991). Inter-relationships between conditioned and primary reinforcement in the maintenance of cigarette smoking. Br J Addict 86: 605-609.

Schultz W (1998). Predictive reward signal of dopamine neurons. J Neurophysiol 80: 1-27.

Shiffman SM, Jarvik ME (1976). Smoking withdrawal symptoms in two weeks of abstinence. Psychopharmacology (Berl) 50: 35-39.

Smith TT, Rupprecht LE, Cwalina SN, Onimus MJ, Murphy SE, Donny EC et al (2016). Effects of monoamine oxidase inhibition on the reinforcing properties of low-dose nicotine. Neuropsychopharmacology 41: 2335-2343.

Sofuoglu M, Herman AI, Nadim H, Jatlow P (2012). Rapid nicotine clearance is associated with greater reward and heart rate increases from intravenous nicotine. Neuropsychopharmacology 37: 1509-1516.

Stead LF, Perera R, Bullen C, Mant D, Lancaster T (2008). Nicotine replacement therapy for smoking cessation (review). Cochrane Database Syst Rev 1: 1-115.

Tang DW, Hello B, Mroziewicz M, Fellows LK, Tyndale RF, Dagher A (2012). Genetic variation in CYP2A6 predicts neural activity to smoking cues as measured using fMRI. Neuroimage 60: 2136-2143.

Tanner JA, Novalen M, Jatlow P, Huestis MA, Murphy SE, Kaprio J et al (2015). Nicotine metabolite ratio (3-hydroxycotinine/ cotinine) in plasma and urine by different analytical methods and laboratories: implications for clinical implementation. Cancer Epidemiol Biomarkers Prev 24: 1239-1246.

Tidey JW, Rohsenow DJ, Kaplan GB, Swift RM, Ahnallen CG (2013). Separate and combined effects of very low nicotine cigarettes and nicotine replacement in smokers with schizophrenia and controls. Nicotine Tob Res 15: 121-129.

Tyndale RF, Sellers EM (2001). Variable CYP2A6-mediated nicotine metabolism alters smoking behavior and risk. Drug Metab Dispos 29: $548-552$.

Watson D, Clark LA, Tellegen A (1988). Development and validation of brief measures of positive and negative affect: the PANAS scales. J Pers Soc Psychol 54: 1063-1072. 